\title{
Constraining CPT violation with Hyper-Kamiokande and ESSnuSB
}

\author{
Rudra Majhi®, ${ }^{1, *}$ Dinesh Kumar Singha ${ }^{1, \dagger}$ K. N. Deepthi, ${ }^{2, \ddagger}$ and Rukmani Mohanta $\oplus^{1, \S}$ \\ ${ }^{1}$ School of Physics, University of Hyderabad, Hyderabad 500046, India \\ ${ }^{2}$ Department of Physics, École Centrale School of Engineering, Mahindra University, \\ Hyderabad 500043, India
}

(Received 29 March 2021; revised 26 July 2021; accepted 28 July 2021; published 7 September 2021)

\begin{abstract}
$C P T$ invariance is one of the most fundamental symmetries in nature and it plays a major role in the formulation of quantum field theory. Although no definitive signal of $C P T$ violation has been observed so far, there are many reasons to carefully investigate various low-energy phenomena that can provide better probes to test $C P T$ symmetry. In this context, neutrino experiments are expected to provide more stringent bounds on $C P T$ invariance violation when compared to the existing bounds from the Kaon system. In this work, we investigate the sensitivity of the upcoming long-baseline experiments: Hyper-Kamiokande (T2HK, T2HKK), ESSnuSB, and DUNE to constrain the $C P T$ violating parameters $\Delta\left(\delta_{C P}\right), \Delta\left(\Delta m_{31}^{2}\right)$, and $\Delta\left(\sin ^{2} \theta_{23}\right)$, which characterize the difference between neutrino and antineutrino oscillation parameters. Further, we analyze neutrino and antineutrino data independently and constrain the oscillation parameters governing them by considering the combination of these experiments (DUNE + T2HKK and DUNE + ESSnuSB). In addition, assuming CPT symmetry is violated in nature, we study the individual ability of the aforementioned experiments to establish $C P T$ violation. We found that the experiments Hyper-Kamiokande (T2HK, T2HKK) and ESSnuSB, along with DUNE, will be able to establish CPT violation in their proposed run-times.
\end{abstract}

DOI: 10.1103/PhysRevD.104.055002

\section{INTRODUCTION}

Understanding the physics beyond the Standard Model $(\mathrm{SM})$ is one of the prime objectives of present-day particle physics research. With the nonobservation of any new heavy beyond the SM particle through direct detection at the LHC, the focus has been shifted to other frontiers, e.g., intensity and cosmic. In the intensity frontier, neutrinos provide a promising avenue for revealing new physics. The compelling evidence of neutrino oscillations from various experiments already indicates that the minimal SM of particle physics is not exhaustive and requires modification. In general, the SM is considered as a low-energy effective theory originating from the unified theory of quantum gravity at the Planck scale. Hence, understanding the true nature of the Planck scale physics through experimental signatures is of great importance, albeit extremely challenging to identify. It is expected that the long-baseline experiments will provide the

\footnotetext{
*rudra.majhi95@gmail.com

dinesh.sin.187@gmail.com

nagadeepthi.kuchibhatla@mahindrauniversity.edu.in

${ }^{\S}$ rmsp@uohyd.ac.in
}

Published by the American Physical Society under the terms of the Creative Commons Attribution 4.0 International license. Further distribution of this work must maintain attribution to the author(s) and the published article's title, journal citation, and DOI. Funded by SCOAP ${ }^{3}$. ideal platform to look for tiny violations of Lorentz invariance or $C P T$ symmetry that may exist as the low-energy remnants of Planck scale physics.

It is well established that local relativistic quantum field theories, including the Standard Model, are invariant under Lorentz and $C P T$ transformations. $C P T$ theorem [1] states that "any quantum theory formulated on flat space time is symmetric under the combined action of $C P T$ transformations, provided the theory respects (i) Locality (ii) Unitarity and (iii) Lorentz invariance." One of the phenomenological consequences of $C P T$ symmetry is that particles and antiparticles will have the same masses and lifetimes. If any discrepancy is found either in their masses or lifetimes, it would be a clear sign of $C P T$ violation. The results from numerous experiments are consistent with the predictions of this symmetry. Although no conclusive evidence of $C P T$ violation has been observed so far, there are many reasons to perform a careful investigation of possible mechanisms and descriptions of Lorentz and $C P T$ violations. One of the ambitious motivations is that the Lorentz and $C P T$ violations might arise from a fundamental theory at the Planck scale but nonetheless may leave their footprints in some low-energy observables that can be detected in the current or upcoming experiments of exceptional sensitivity.

Studies related to $C P T$ violation are not new, see, e.g., Refs. [2-19]. There are several theories by which quantum gravity induced $C P T$ violation can occur. Especially 
neutrinos, in addition to neutral kaons [20], make potential candidates to provide good insight into $C P T$ violation, if it exists. For instance, some interesting aspects of quantum gravity decoherence (nonlocal) in neutrinos introduce $C P T$ violation and account for the smallness of neutrino mass [21].

There exist experimental limits on $C P T$ violating parameters from kaon and the lepton sectors. However, the current neutrino oscillation data provides the most stringent constraints on various oscillation parameters [17]:

$$
\begin{aligned}
\left|\Delta m_{21}^{2}-\Delta \bar{m}_{21}^{2}\right| & <4.7 \times 10^{-5} \mathrm{eV}^{2}, \\
\left|\Delta m_{31}^{2}-\Delta \bar{m}_{31}^{2}\right| & <2.5 \times 10^{-4} \mathrm{eV}^{2}, \\
\left|\sin ^{2} \theta_{12}-\sin ^{2} \bar{\theta}_{12}\right| & <0.14, \\
\left|\sin ^{2} \theta_{13}-\sin ^{2} \bar{\theta}_{13}\right| & <0.029, \\
\left|\sin ^{2} \theta_{23}-\sin ^{2} \bar{\theta}_{23}\right| & <0.19 .
\end{aligned}
$$

Further, in Ref. [3] it has been shown that DUNE will test the $C P T$ violation in atmospheric mass-squared difference to an unprecedented level and provide the most stringent limit as $\left|\Delta m_{31}^{2}-\Delta \bar{m}_{31}^{2}\right|<8.1 \times 10^{-5} \mathrm{eV}^{2}$ at $3 \sigma$ C.L.

Without delving into the specifications of any model, in this work we would like to test the predictions of $C P T$ conservation in the light of future neutrino oscillation experiments Hyper-Kamiokande (T2HK and T2HKK), the European Spallation Source Neutrino Super Beam (ESSnuSB) project, and Deep Underground Neutrino Experiment (DUNE). Since neutrino oscillation experiments are only sensitive to mass-squared differences and mixing angles, one can test the CPT symmetry by measuring the differences in the oscillation parameters of neutrinos and antineutrinos. If the fundamental $C P T$ invariance is not assumed, neutrinos and antineutrinos need to be parametrized by different $3 \times 3$ unitary mixing matrices. In the case of neutrinos, the flavor eigenstates $\left|\nu_{\alpha}\right\rangle$ and the mass eigenstates $\left|\nu_{i}\right\rangle$ are related by a $3 \times 3$ unitary leptonic mixing matrix [22],

$$
\left|\nu_{\alpha}\right\rangle=\sum_{i=1}^{3} U_{\alpha i}\left(\theta_{12}, \theta_{13}, \theta_{23}, \delta_{C P}\right)\left|\nu_{i}\right\rangle .
$$

Analogously, the corresponding states for the antineutrinos are related as

$$
\left|\bar{\nu}_{\alpha}\right\rangle=\sum_{i=1}^{3} U_{\alpha i}^{*}\left(\bar{\theta}_{12}, \bar{\theta}_{13}, \bar{\theta}_{23}, \bar{\delta}_{C P}\right)\left|\bar{\nu}_{i}\right\rangle .
$$

Denoting the neutrino and antineutrino masses by $m_{i}$ and $\bar{m}_{i}(i=1,2,3)$, the mass-squared differences of neutrinos are represented as $\Delta m_{i j}^{2} \equiv m_{i}^{2}-m_{j}^{2}$ and that of antineutrinos as $\Delta \bar{m}_{i j}^{2} \equiv \bar{m}_{i}^{2}-\bar{m}_{j}^{2}$. Consequently the oscillation probabilities of neutrinos and antineutrinos are functions of the oscillation parameters $\left(\theta_{12}, \theta_{13}, \theta_{23}, \Delta m_{21}^{2}, \Delta m_{31}^{2}, \delta_{C P}\right)$ and $\left(\bar{\theta}_{12}, \bar{\theta}_{13}, \bar{\theta}_{23}, \Delta \bar{m}_{21}^{2}, \Delta \bar{m}_{31}^{2}, \bar{\delta}_{C P}\right)$, respectively. In principle, neutrino oscillation experiments will be able to place bounds on the predictions of $C P T$ symmetry violation. In this work, we investigate the ability of the future longbaseline experiments: T2HK, T2HKK, ESSnuSB, and DUNE to constrain the $C P T$ violating parameters, such as $\left|\delta_{C P}-\bar{\delta}_{C P}\right|,\left|\Delta m_{31}^{2}-\Delta \bar{m}_{31}^{2}\right|$, and $\left|\sin ^{2} \theta_{23}-\sin ^{2} \bar{\theta}_{23}\right|$. We further analyze neutrino and antineutrino data independently and constrain the oscillation parameters by considering the combination of the experiments DUNE + T2HKK and DUNE + ESSnuSB. In addition, assuming $C P T$ symmetry is violated in nature, we study the individual ability of T2HK, T2HKK, DUNE, and ESSnuSB experiments to establish $C P T$ violation.

The outline of the paper is as follows. In Sec. II, we give a brief overview of the experimental and simulation details of T2HK, T2HKK, ESSnuSB, and DUNE. In Sec. III, we determine the bounds placed by these experiments on the parameters $\Delta\left(\delta_{C P}\right), \Delta\left(\Delta m_{31}^{2}\right)$, and $\Delta\left(\sin ^{2} \theta_{23}\right)$ by assuming $C P T$ symmetry exists in nature. Further, in Sec. III A we analyze the combined data of DUNE + T2HKK, DUNE + ESSnuSB and evaluate how well they can measure neutrino and antineutrino oscillation parameters independently. Additionally, in Sec. IV we assume that $C P T$ symmetry is violated in nature and estimate the sensitivity of T2HK, T2HKK, ESSnuSB, and DUNE to establish $C P T$ invariance violation individually. Finally, our results are summarized in Sec. V.

\section{EXPERIMENTAL AND SIMULATION DETAILS}

In this section, we discuss the detailed experimental features of the long-baseline experiments T2HK, T2HKK, ESSnuSB, and DUNE.

Tokai to Hyper-Kamiokande (T2HK) is an upgradation proposed to the existing T2K facility in Japan. In this plan, the JPARC beam will produce a 1.3 MW powered beam and the far detector (FD) will have two identical water Cherenkov detectors of $187 \mathrm{kt}(2 \times 187=374 \mathrm{kt})$ fiducial volume to be placed at $295 \mathrm{~km}$ baseline, $2.5^{\circ}$ off from the beam axis.

T2HKK is an alternative choice to T2HK, where the proposed FD is placed in Korea, which is $1100 \mathrm{~km}$ away from the JPARC facility. One of the two tanks (187 kt) proposed in the T2HK experiment will be placed at $1100 \mathrm{~km}$ with an off-axis angle (OAA) of $1.5^{\circ}$ or $2^{\circ}$ or $2.5^{\circ}$. Basing on the study in [23], we consider the off-axis angle $1.5^{\circ}$ as it provides maximum sensitivity to the oscillation parameters. We consider the proposed run time ratio of $(1 \nu: 3 \bar{\nu})$ years corresponding to a total exposure of $27 \times 10^{21}$ protons on target. The detector systematics are taken as per those of $[23,24]$.

The major objective of the ESSnuSB project [25] is to measure the leptonic $C P$ violation. A neutrino beam with a peak energy of $0.25 \mathrm{GeV}$ is produced at the ESS facility in 
TABLE I. The experimental specifications and systematic uncertainties of T2HK, T2HKK, ESSnuSB, and DUNE.

\begin{tabular}{lcccc}
\hline \hline Experiment & T2HK & T2HKK & ESSnuSB & DUNE \\
\hline Baseline & $295 \mathrm{~km}$ & $295 \mathrm{~km} ; 1100 \mathrm{~km}$ & $540 \mathrm{~km}$ & $1300 \mathrm{~km}$ \\
Fiducial volume & $374 \mathrm{kt}$ & $187 \mathrm{kt}(@ 295 \mathrm{~km})+187 \mathrm{kt}(1100 \mathrm{~km})$ & $500 \mathrm{kt}$ & $40 \mathrm{kt}$ \\
Normalization uncertainty & & & & \\
$\nu_{e}$ signal (bkg) & $3.2 \%(5 \%)$ & $3.8 \%(5 \%)$ & $3.2 \%(5 \%)$ & $2 \%(5 \%)$ \\
$\bar{\nu}_{e}$ signal (bkg) & $3.9 \%(5 \%)$ & $4.1 \%(5 \%)$ & $3.9 \%(5 \%)$ & $2 \%(5 \%)$ \\
$\nu_{\mu}$ signal (bkg) & $3.6 \%(5 \%)$ & $3.8 \%(5 \%)$ & $3.6 \%(5 \%)$ & $5 \%(5 \%)$ \\
$\bar{\nu}_{\mu}$ signal (bkg) & $3.6 \%(5 \%)$ & $3.8 \%(5 \%)$ & $3.6 \%(5 \%)$ & $5 \%(5 \%)$ \\
\hline \hline
\end{tabular}

Lund, Sweden. This beam is made to travel $540 \mathrm{~km}$ to encounter a water Cherenkov detector of $500 \mathrm{kt}$ to be placed at a mine in Garpenberg. The proposed runtime is $(2 \nu+8 \bar{\nu})$ years with total protons on target of $27 \times 10^{22}$ corresponding to a $5 \mathrm{MW}$ proton beam.

DUNE [26] comprises of a broad band neutrino beam of $0.5-8 \mathrm{GeV}$ energy, a near detector at Fermilab, and a liquid argon time projection chamber of fiducial volume $40 \mathrm{kt}$ located at $1300 \mathrm{~km}$ in South Dakota. We have considered $(5 \nu+5 \bar{\nu})$ year run-time, beam power of $1.2 \mathrm{MW}$ corresponding to $10 \times 10^{21}$ protons on target.

We have performed the numerical analysis using the GLoBES package [27,28]. The experimental specifications, along with the signal and background normalization errors, are listed in Table I.

The statistical $\chi^{2}$ is obtained using

$$
\chi_{\text {stat }}^{2}=2 \sum_{i}\left\{N_{i}^{\text {test }}-N_{i}^{\text {true }}+N_{i}^{\text {true }} \ln \frac{N_{i}^{\text {true }}}{N_{i}^{\text {test }}}\right\}
$$

where $N_{i}^{\text {test }}$ corresponds to the number of events predicted by the model while $N_{i}^{\text {true }}$ denotes the total number of simulated events (signal and background) in $i$ th bin. The systematic uncertainties are incorporated into the simulation using the Pull method. The pull variables being the signal and background normalization uncertainties of $\nu_{e}, \bar{\nu}_{e}$ appearance and $\nu_{\mu}, \bar{\nu}_{\mu}$ disappearance channels. The values of the normalization errors on signals and backgrounds (bkg) corresponding to different channels of the experiments are listed in Table I. Here, $\chi_{\text {pull }}^{2}$ accounts for these errors and acts as a penalty term to the total $\chi^{2}\left(\chi_{\mathrm{tot}}^{2}\right)$.

\section{SENSITIVITY OF THE EXPERIMENTS T2HK, T2HKK, ESSnuSB, TO CPT VIOLATION}

In this section, we calculate the $C P T$ violation sensitivity of the long-baseline experiments T2HK, T2HKK, ESSnuSB, and DUNE. At first, we assume that there is no intrinsic $C P T$ violation in nature, i.e., both neutrino and antineutrino parameters are equal. That is, for a given oscillation parameter, we simulate the data for each of these experiments with $\Delta x=|x-\bar{x}|=0$, where $x(\bar{x})$ is the oscillation parameter for neutrinos (antineutrinos). Then we evaluate the sensitivity of each of the experiments to nonzero $\Delta x$. The true values for oscillation parameters used are given in Table II. In each case, we choose three values for $\theta_{23}$ : lower octant $\left(\sin ^{2} \theta_{23}=0.43\right)$, maximal $\left(\sin ^{2} \theta_{23}=0.5\right)$, and higher octant $\left(\sin ^{2} \theta_{23}=0.57\right)$ to study the correlation between the $C P T$ sensitivity and the octant of $\theta_{23}$. In the test values, we marginalize over all the oscillation parameters for both neutrinos and antineutrinos except $x, \bar{x}$ and the solar parameters (since T2HK, T2HKK, ESSnuSB, and DUNE have no sensitivity to these parameters). After marginalization, we calculate the $\chi^{2}$ value for the $C P T$ violating observable through the relation

$$
\chi^{2}(\Delta x)=\chi^{2}(|x-\bar{x}|)=\chi^{2}(x)+\chi^{2}(\bar{x})
$$

and the minimum $\chi^{2}(\Delta x)$ has been calculated over all possible combinations of $|x-\bar{x}|$.

In Figs. 1-3, we show the $C P T$ violation sensitivities of these experiments to oscillation parameters $\Delta\left(\Delta m_{31}^{2}\right) \equiv$ $\Delta m_{31}^{2}-\Delta \bar{m}_{31}^{2}, \quad \Delta\left(\sin ^{2} \theta_{23}\right) \equiv \sin ^{2} \theta_{23}-\sin ^{2} \bar{\theta}_{23}, \quad$ and $\Delta\left(\delta_{C P}\right) \equiv \delta_{C P}-\bar{\delta}_{C P}$, respectively. The results in left, middle, and right columns of these figures are obtained by assuming the octant of $\theta_{23}$ as low, maximal, and high, respectively. The colored curves blue, red, green, and magenta show the sensitivities of T2HK, T2HKK, ESSnuSB, and DUNE, respectively. The black dash-dot line represents $3 \sigma$ confidence limit. The sensitivity for $\Delta\left(\sin ^{2} \theta_{13}\right)$ is not very significant, for which we have not shown the corresponding result here.

TABLE II. The values of oscillation parameters that we considered in our analysis are taken from Ref. [29].

\begin{tabular}{lcc}
\hline \hline Parameters & True values & Test value range \\
\hline $\sin ^{2} \theta_{12}$ & 0.304 & $\mathrm{NA}$ \\
$\sin ^{2} \theta_{13}$ & 0.02221 & $0.02034 \rightarrow 0.02430$ \\
$\sin ^{2} \theta_{23}$ & $0.43,0.5,057$ & $0.4 \rightarrow 0.62$ \\
$\delta_{C P}$ & $195^{\circ}$ & $0^{\circ} \rightarrow 360^{\circ}$ \\
$\Delta m_{12}^{2}$ & $7.42 \times 10^{-5} \mathrm{eV}^{2}$ & $\mathrm{NA}$ \\
$\Delta m_{31}^{2}$ & $2.514 \times 10^{-3} \mathrm{eV}^{2}(\mathrm{Normal}$ & $(2.431 \rightarrow 2.598)$ \\
& Hierarchy) & $\times 10^{-3} \mathrm{eV}^{2}$ \\
\hline \hline
\end{tabular}



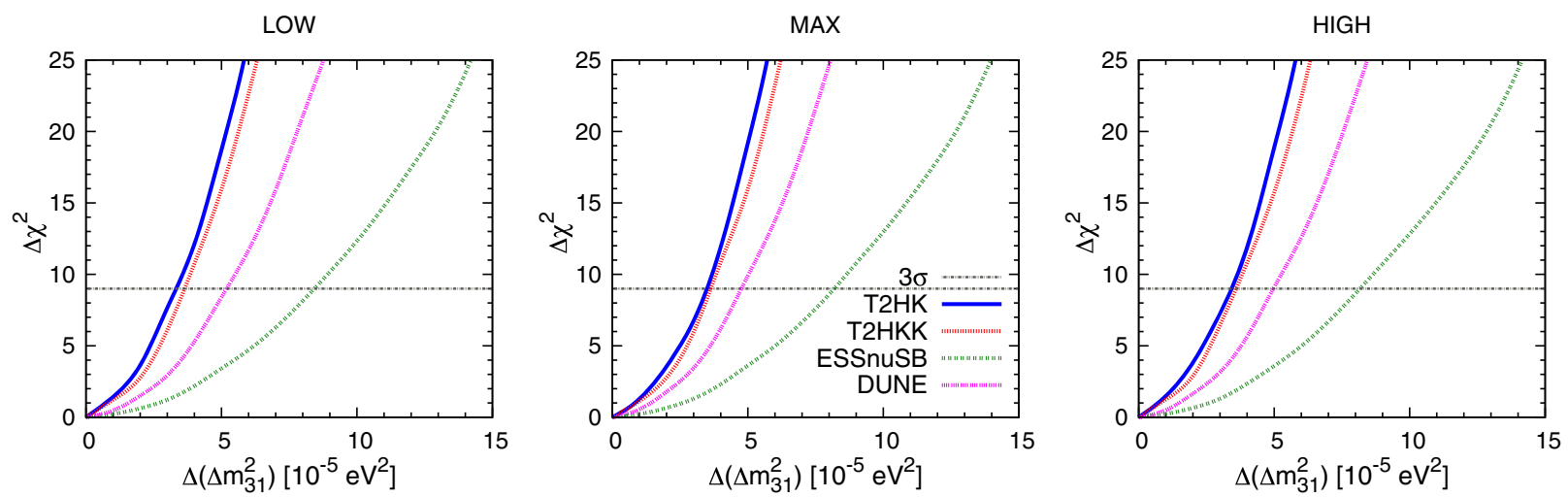

FIG. 1. The sensitivity of the experiments T2HK (blue curve), T2HKK (red curve), ESSnuSB (green curve), and DUNE (magenta curve) to $\Delta\left(\Delta m_{31}^{2}\right)$.
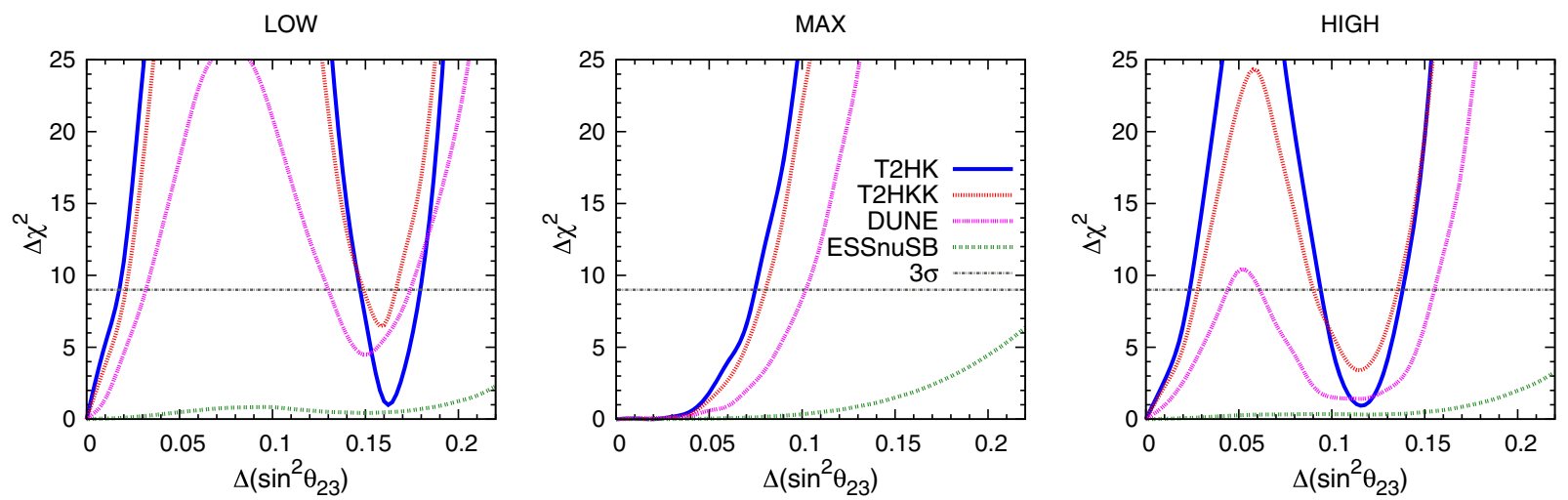

FIG. 2. The sensitivity of the experiments T2HK (blue curve), T2HKK (red curve), ESSnuSB (green curve), and DUNE (magenta curve) to $\Delta\left(\sin ^{2} \theta_{23}\right)$.

From Fig. 1, one can estimate the best bound on the parameter $\Delta\left(\Delta m_{31}^{2}\right)$ at $3 \sigma$ C.L. by T2HK experiment (blue curve) as $\Delta\left(\Delta m_{31}^{2}\right)<3.32 \times 10^{-5} \mathrm{eV}^{2}$. It can be seen from all the columns of Fig. 1 that T2HK provides a better bound compared to T2HKK, ESSnuSB, and DUNE, for all the three values of $\theta_{23}$ considered. The alternative choice of the
Hyper-Kamiokande experiment, i.e., T2HKK (red curve) provides better bound on $\Delta\left(\Delta m_{31}^{2}\right)<3.62 \times 10^{-5} \mathrm{eV}^{2}$ at $3 \sigma$ C.L. than the bound from DUNE (magenta curve) experiment obtained in Ref. [3] and ESSnuSB. The list of the bounds at $3 \sigma$ C.L. on $\Delta\left(\Delta m_{31}^{2}\right)$ for $\theta_{23}<45^{\circ}, \theta_{23}=45^{\circ}$ and $\theta_{23}>45^{\circ}$ are given in the first row of Table III. Here it is
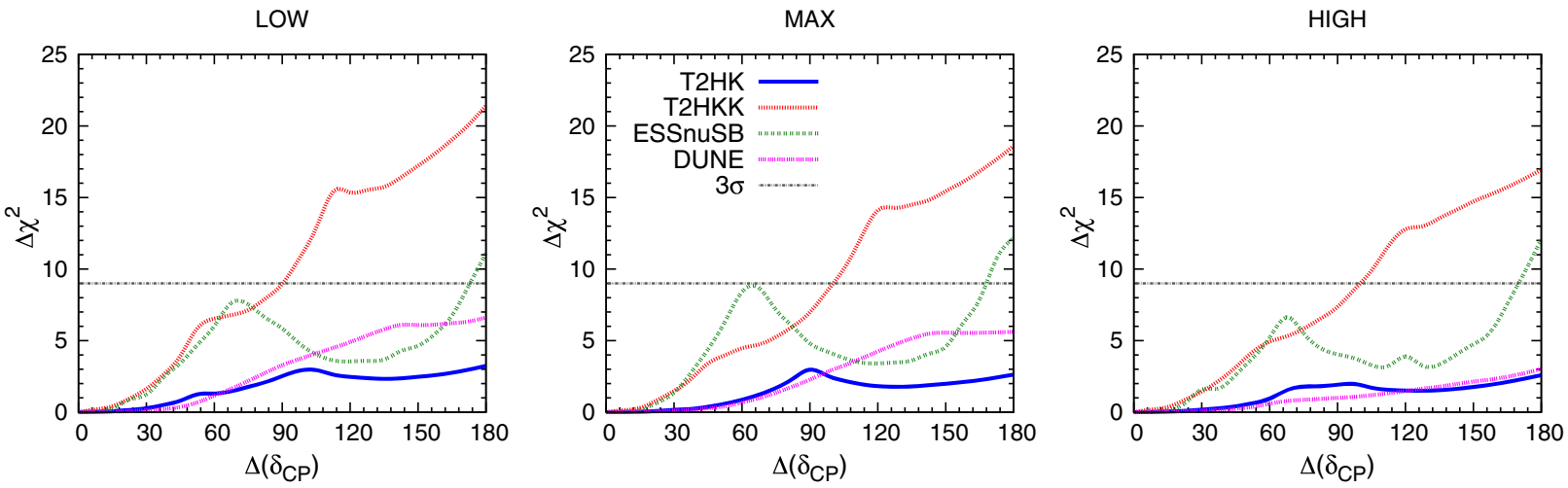

FIG. 3. The sensitivity of the experiments T2HK (blue curve), T2HKK (red curve), ESSnuSB (green curve), and DUNE (magenta curve) to $\Delta\left(\delta_{C P}\right)$. 
TABLE III. Bounds on the parameters at $3 \sigma$ C.L. from T2HK, T2HKK, ESSnuSB, and DUNE experiments. The set of three values in the brackets correspond to the results for $\theta_{23}$ value as $\theta_{23}<45^{\circ}, \theta_{23}=45^{\circ}$ and $\theta_{23}>45^{\circ}$.

\begin{tabular}{lcccc}
\hline \hline Parameters & T2HK & T2HKK & ESSnuSB & DUNE \\
\hline$\Delta\left(\Delta m_{31}^{2}\right)\left[10^{-5} \mathrm{eV}^{2}\right]$ & {$[3.32,3.45,3.4]$} & {$[3.62,3.62,3.59]$} & {$[8.43,8.18,8.14]$} & {$[5.2,4.77,4.96]$} \\
$\Delta\left(\sin ^{2} \theta_{23}\right)$ & {$[0.180,0.075,0.139]$} & {$[0.167,0.08,0.135]$} & $\ldots$ & {$[0.173,0.102,0.155]$} \\
$\Delta\left(\delta_{C P}\right)$ & $\cdots$ & {$[90,100,100]^{\circ}$} & {$[173,168,170]^{\circ}$} & $\ldots$ \\
\hline \hline
\end{tabular}

important to note that these experiments will highly improve upon the existing bounds posed by neutral kaon system.

It can be noted from the three plots of Fig. 2 that different sensitivities to $\Delta\left(\sin ^{2} \theta_{23}\right)$ are obtained for different true values of $\theta_{23}$. First, when the true value of $\theta_{23}$ is in higher and lower octants, degenerate solutions are obtained for $\Delta\left(\sin ^{2} \theta_{23}\right)$ at $3 \sigma$ C.L. in the complementary octant for all the four experiments. However, for lower octant of $\theta_{23}$, this degeneracy does not exist in the case of T2HKK (red) and DUNE (magenta) at $2 \sigma$ C.L. Second, for true maximal $\theta_{23}$, the sensitivity of the experiments increase with the increasing values of $\Delta\left(\sin ^{2} \theta_{23}\right)$. Furthermore, ESSnuSB provides comparatively very low $C P T$ violation sensitivity to $\Delta\left(\sin ^{2} \theta_{23}\right)$ for all values of true $\theta_{23}$. The list of the bounds at $3 \sigma$ C.L. on $\Delta\left(\sin ^{2} \theta_{23}\right)$ are given in the second row of Table III.

From Fig. 3 , the best ever bounds on $\Delta\left(\delta_{C P}\right)$ can be extracted from T2HKK (red curve) for $C P T$ violation which is $\Delta\left(\delta_{C P}\right)<100^{\circ}$ at $3 \sigma$ confidence level. The next best bound on $\Delta\left(\delta_{C P}\right)$ is obtained by ESSnuSB experiment. This is because both T2HKK and ESSnuSB experiment are planned at the second oscillation maxima to meet their primary goal of measuring the $C P$ phase $\delta_{C P}$. The list of the bounds at $3 \sigma$ C.L. on $\Delta\left(\delta_{C P}\right)$ are given in the third row of Table III.

\section{A. Constraining $C P T$ violation with combination of DUNE + T2HKK and DUNE + ESSnuSB}

In this subsection, we continue to assume that $C P T$ is a conserved symmetry in nature. We analyze the neutrino and antineutrino data independently and determine whether the corresponding oscillation parameters in both cases are the same as predicted by $C P T$ symmetry. The true oscillation parameters that are considered in the analysis are provided in Table II and for the test scenario, we take the six oscillation parameters for both neutrino $\left(\Delta m_{31}^{2}, \theta_{23}, \delta_{C P}\right)$ and antineutrino $\left(\Delta \bar{m}_{31}^{2}, \bar{\theta}_{23}, \bar{\delta}_{C P}\right)$ in their allowed ranges as given in Table II. Marginalization is done over the remaining four parameters while showing the effect of the rest two oscillation parameters. The results are shown in Fig. 4, where the axes can be visualized for both neutrino and antineutrino parameters. It is shown in Ref. [30] that, while DUNE and T2HK can resolve the octant degeneracy assuming CPT conservation, the combination of DUNE+ T2HK cannot resolve this degeneracy while treating neutrino and antineutrino parameters individually. In this subsection, we explore the same by considering the combination of DUNE + T2HKK and DUNE + ESSnuSB experiments. The blue and red contours in all the plots in Fig. 4 represent the allowed contours with 99\% C.L. for neutrino and antineutrino data, respectively. The left (right) panel shows the allowed regions of the neutrino and antineutrino oscillation parameters of DUNE + T2HKK (DUNE + ESSnuSB) experiments. From the middle and bottom plots of left panel, it can be seen that DUNE + T2HKK resolve octant degeneracy in $\theta_{23}$, and $\bar{\theta}_{23}$ at 99\% C.L. when CPT conservation is assumed in nature. Besides, when we combined the simulated data from the antineutrino beams of DUNE and ESSnuSB (red curves of right side panel) degenerate solutions to $\delta_{C P}$ and $\theta_{23}$ are obtained. However, this degeneracy disappears when we considered the neutrino beams of DUNE and ESSnuSB. Overall, from all the plots of Fig. 4, we can observe that neutrino oscillation data constrains the parameters better than the antineutrino data.

\section{DISCOVERING CPT VIOLATION}

In this section, we assume that $C P T$ is violated in nature. We generate the simulated data for the experiments T2HK, T2HKK, ESSnuSB, and DUNE by assuming different neutrino and antineutrino oscillation parameters. In particular, we only consider the case where the $C P$ violating phases $\delta_{C P}$ and $\bar{\delta}_{C P}$ are not equal. ${ }^{1}$ We further consider $\theta_{23}=\bar{\theta}_{23}, \Delta m_{31}^{2}=\Delta \bar{m}_{31}^{2}, \theta_{13}=\bar{\theta}_{13}$, and their true values are taken from Table II. In Fig. 5, we plot the allowed regions of $\delta_{C P}$ (test) and $\bar{\delta}_{C P}($ test $)$ as obtained from the experiments T2HK (blue), T2HKK (red), ESSnuSB (green), and DUNE (magenta). The solid and dotted contours in all the figures correspond to $68 \%$ and 99\% C.L. and the dashed black lines correspond to $C P T$ conserving values. Top panel of Fig. 5 shows that both the configurations of Hyper-Kamiokande experiment-T2HK (blue) and T2HKK (red) will be able to establish CPT violation with $99 \%$ C.L. by showing that $\delta_{C P} \neq \bar{\delta}_{C P}$ in their proposed run-time. This can be inferred from the fact that there are no degenerate solutions obtained in the figure. However, T2HKK provides tighter constraints on the parameter space of $\delta_{C P}-\bar{\delta}_{C P}$, compared with T2HK, as

\footnotetext{
${ }^{1}$ We consider the variation in $\delta_{C P}$ and $\bar{\delta}_{C P}$ as these parameters are poorly constrained.
} 
DUNE+T2HKK

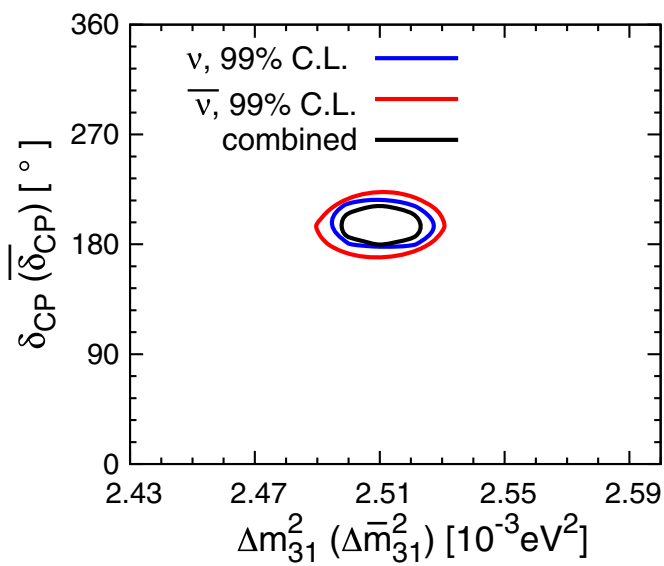

DUNE+T2HKK

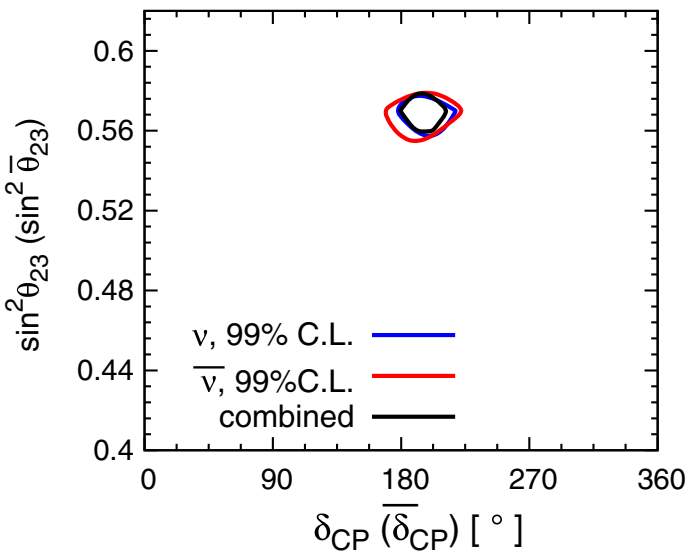

DUNE+T2HKK

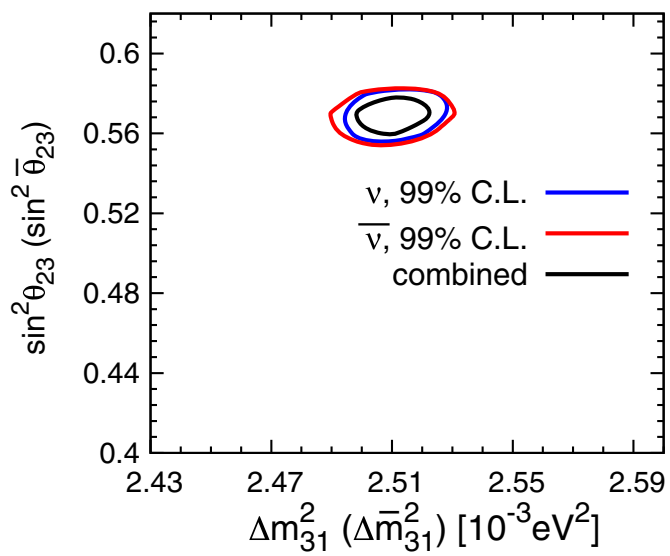

DUNE+ESSnuSB

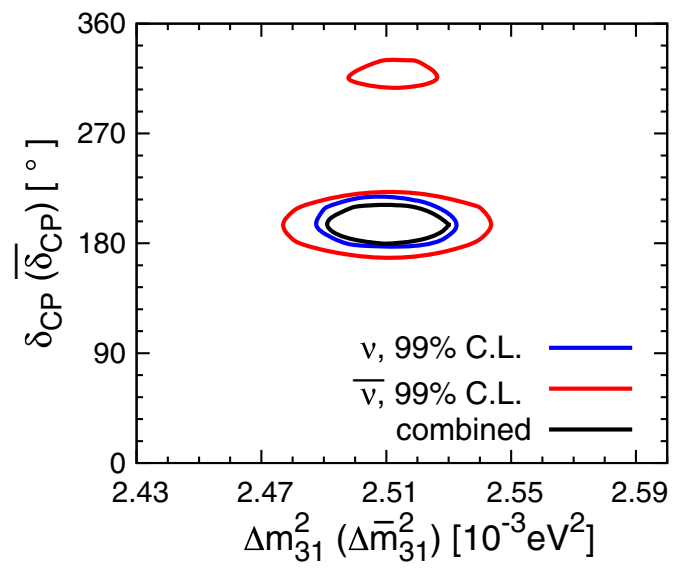

DUNE+ESSnuSB

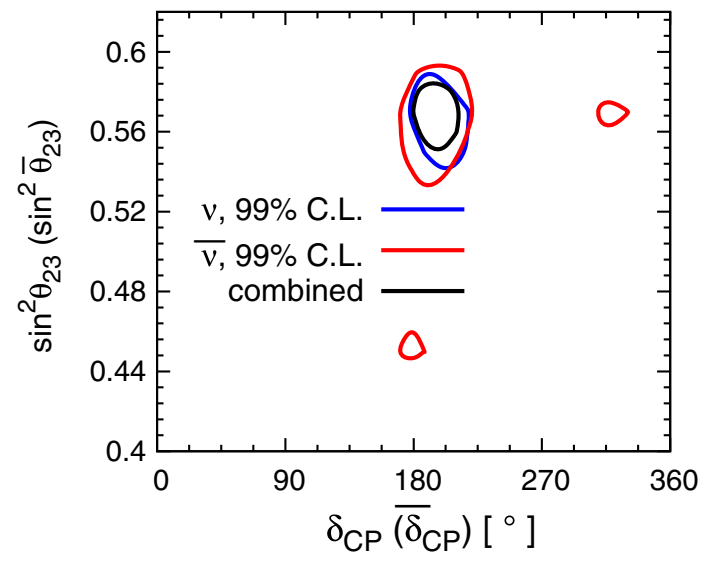

DUNE+ESSnuSB

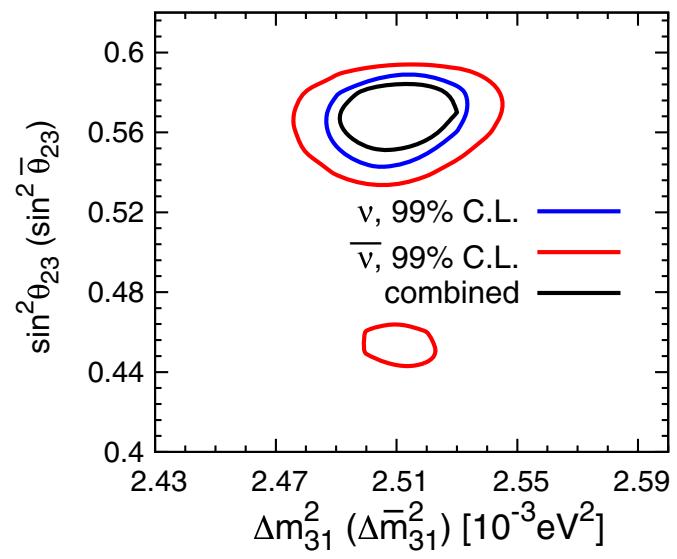

FIG. 4. Allowed parameter space between different neutrino and antineutrino oscillation parameters at $99 \%$ C.L. for combination of DUNE and T2HKK as well as DUNE and ESSnuSB experiments. In each plot, the blue (red) curve is for neutrino (antineutrino) parameters and the black curve is the combined result of neutrino and antineutrino parameters.

seen from the red and blue contours of the top panel. This can be attributed to the higher sensitivity of T2HKK experiment to the $C P$ violating phase $\delta_{C P}$ as its FD (Korea) is going to be placed at second oscillation maxima $\left(\nu_{\mu} \rightarrow \nu_{e}\right.$ channel) unlike in the case of the T2HK experiment, which focuses on first oscillation maxima. The bottom panel of Fig. 5 shows that ESSnuSB and DUNE can establish CPT violation with $99 \%$ C.L. on their own. Additionally, since ESSnuSB experiment has higher sensitivity to $C P$ phase when compared to DUNE, it can be 

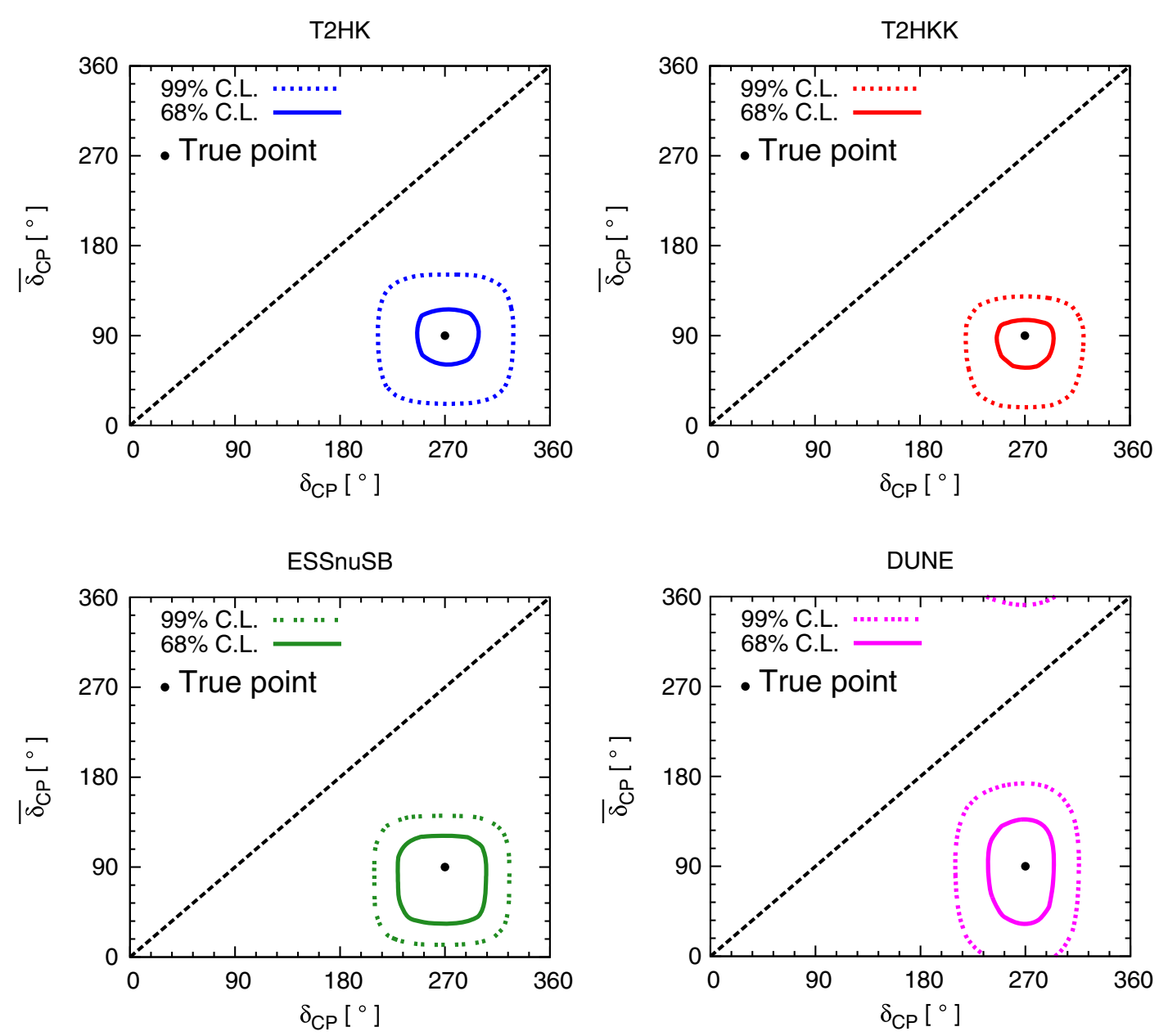

FIG. 5. Allowed regions between $\delta_{C P}$ and $\bar{\delta}_{C P}$ in the $C P T$ violating scenario. Solid (dotted) curve shows the parameter space at $68 \%$ and $99 \%$ C.L.

seen that the green contours (ESSnuSB) in the bottom left panel are tighter than the magenta contours (DUNE) of the bottom right panel. In conclusion, if nature has $C P T$ violation, then the forthcoming experiments HyperKamiokande, ESSnuSB, and DUNE will be able to establish $C P T$ violation individually in their proposed run-times by establishing that $\delta_{C P} \neq \bar{\delta}_{C P}$.

\section{SUMMARY}

$C P T$ symmetry is one of the fundamental symmetries of nature and its breaking is related to Planck scale physics. However, $C P T$ violation has not been observed unambiguously so far, and it is an exciting challenge to search for its implications. It is expected that some of the $C P T$ violating observables might be observed at low-energy scales. So far, the most stringent bound on $C P T$ violation comes from the neutral kaon sector. Remarkably, the current evidence for neutrino oscillations lies at levels where Planck-suppressed effects might be expected to appear. Hence, the neutrino sector can provide a better opportunity to explore $C P T$ violation.
In this paper, we have studied the sensitivity reach of the upcoming long-baseline experiments, T2HK, T2HKK, ESSnuSB, and DUNE to explore the CPT violation in the neutrino sector. Our findings are summarized below:

(1) We obtained the sensitivity limits on the $C P T$ violating parameters $\Delta\left(\Delta m_{31}^{2}\right), \Delta\left(\sin ^{2} \theta_{23}\right)$, and $\Delta\left(\delta_{C P}\right)$. We found that the T2HKK and ESSnuSB experiments are quite sensitive to the $C P$ violating phase $\delta_{C P}$, whereas T2HK, T2HKK, and DUNE are sensitive to the atmospheric mixing parameters. The most stringent limits on $\Delta\left(\Delta m_{31}^{2}\right)$ and $\Delta\left(\sin ^{2} \theta_{23}\right)$ come from T2HK experiment, whereas T2HKK will provide the best bound on $\Delta\left(\delta_{C P}\right)$.

(2) Next, we obtained the constraint on $C P T$ violation with the combination DUNE + T2HKK and DUNE + ESSnuSB experiments. Assuming that nature is invariant under $C P T$, we analyzed the neutrino and antineutrino data independently for these combinations of experiments and scrutinized whether they provide the same oscillation parameters as predicted by $C P T$ symmetry. We found that these experiments are sensitive to $C P T$ violation and 
DUNE + T2HKK can even resolve the octant degeneracy in $\theta_{23}$ and $\bar{\theta}_{23}$ at $99 \%$ C.L.

(3) Finally, we have shown that if $C P T$ violation exists in nature, the upcoming long-baseline experiments T2HK, T2HKK, ESSnuSB, and DUNE will be able to establish $C P T$ violation individually at $99 \%$ C.L. in their proposed run-times by demonstrating $\delta_{C P} \neq \bar{\delta}_{C P}$. In conclusion, we found that the upcoming experiments T2HK, T2HKK, ESSnuSB, and DUNE have great potential to establish $C P T$ violation in neutrino oscillation and provide stringent limits on the $C P T$ violating parameters $\Delta\left(\Delta m_{31}^{2}\right)$ and $\Delta\left(\sin ^{2} \theta_{23}\right)$.

\section{ACKNOWLEDGMENTS}

The authors would like to thank Enrique FernandezMartinez for sharing the ESSnuSB GLoBES files. One of the authors (R. M.) would like to thank the DST-INSPIRE program for financial support. D. K. S. acknowledges CSIR, Government of India, for financial support. The work of R. M. is supported in part by SERB, Government of India through Grant No. EMR/2017/001448 and University of Hyderabad IoE project Grant No. RC1-20012. We gratefully acknowledge the use of CMSD HPC facility of University of Hyderabad to carry out computations in this work.
[1] G. Luders, Ann. Phys. (N.Y.) 2, 1 (1957).

[2] V. A. Kostelecky and M. Mewes, Phys. Rev. D 70, 031902 (2004).

[3] G. Barenboim, C. A. Ternes, and M. Tortola, Phys. Lett. B 780, 631 (2018).

[4] V. A. Kostelecky and M. Mewes, Phys. Rev. D 69, 016005 (2004).

[5] H. Minakata and S. Uchinami, Phys. Rev. D 72, 105007 (2005).

[6] A. de Gouvea and Y. Grossman, Phys. Rev. D 74, 093008 (2006).

[7] J. S. Diaz, V. A. Kostelecky, and M. Mewes, Phys. Rev. D 80, 076007 (2009).

[8] J. S. Diaz and A. Kostelecky, Phys. Rev. D 85, 016013 (2012).

[9] A. Kostelecky and M. Mewes, Phys. Rev. D 85, 096005 (2012).

[10] J. S. Diaz, T. Katori, J. Spitz, and J. M. Conrad, Phys. Lett. B 727, 412 (2013).

[11] A. Chatterjee, R. Gandhi, and J. Singh, J. High Energy Phys. 06 (2014) 045.

[12] T. Ohlsson and S. Zhou, Nucl. Phys. B893, 482 (2015).

[13] M. Jacobson and T. Ohlsson, Phys. Rev. D 69, 013003 (2004).

[14] K. Abe et al. (Super-Kamiokande Collaboration), Phys. Rev. D 91, 052003 (2015).

[15] C. A. Arguelles, T. Katori, and J. Salvado, Phys. Rev. Lett. 115, 161303 (2015).
[16] J. S. Diaz and T. Schwetz, Phys. Rev. D 93, 093004 (2016).

[17] G. Barenboim, C. A. Ternes, and M. Tortola, J. High Energy Phys. 07 (2020) 155.

[18] R. Majhi, C. Soumya, and R. Mohanta, Eur. Phys. J. C 80, 364 (2020).

[19] Daljeet Kaur, Phys. Rev. D 101, 055017 (2020).

[20] B. Schwingenheuer et al., Phys. Rev. Lett. 74, 4376 (1995).

[21] A. Capolupo, S. M. Giampaolo, and G. Lambiase, Phys. Lett. B 792, 298 (2019).

[22] S. M. Bilenky and S. T. Petcov, Rev. Mod. Phys. 59, 671 (1987); 60, 575(E) (1988); 61, 169(E) (1989).

[23] K. Abe et al. (Hyper-Kamiokande Proto-Collaboration), Prog. Theor. Exp. Phys. 2018, 063 C01 (2018).

[24] K. Abe et al. (Hyper-Kamiokande Proto-Collaboration), arXiv:1805.04163.

[25] E. Baussan et al., Nucl. Phys. B885, 127 (2014).

[26] R. Acciarri et al. (DUNE Collaboration), arXiv:1512.06148.

[27] P. Huber, M. Lindner, and W. Winter, Comput. Phys. Commun. 167, 195 (2005).

[28] P. Huber, J. Kopp, M. Lindner, M. Rolinec, and W. Winter, Comput. Phys. Commun. 177, 432 (2007).

[29] I. Esteban, M. C. Gonzalez-Garcia, M. Maltoni, T. Schwetz, and A. Zhou, J. High Energy Phys. 09 (2020) 178.

[30] A. de Gouvea and K. J. Kelly, Phys. Rev. D 96, 095018 (2017). 